\title{
EL PSICOTERAPEUTA EN EL PROCESO TERAPÉUTICO
}

\section{THE PSYCHOTHERAPIST IN THE THERAPEUTIC PROCESS}

\author{
Sergi Corbella
}

FPCEE Blanquerna-Universitat Ramon Llull. Barcelona, España

ORCID: https://orcid.org/0000-0003-1858-2988

Cómo referenciar este artículo/How to reference this article:

Corbella, S. (2020). El psicoterapeuta en el proceso terapéutico. Revista de Psicoterapia, 31(116), 91103. https://doi.org/10.33898/rdp.v31i116.409

\begin{abstract}
Resumen
El psicoterapeuta es uno de los protagonistas del proceso terapéutico. Son muchos los esfuerzos dirigidos a estudiar los factores que influyen en el establecimiento de una buena relación entre psicoterapeuta y paciente que permita lograr un buen resultado psicoterapéutico. En el artículo se profundiza en las características, habilidades y acciones del psicoterapeuta en el contexto de la relación de ayuda del proceso psicoterapéutico. Se plantean algunos principios y retos para contribuir y promover la mejora continua en nuestra práctica profesional como psicoterapeutas.

Palabras clave: Psicoterapeuta, proceso terapéutico, alianza terapéutica, estilo personal del terapeuta.
\end{abstract}

\begin{abstract}
The psychotherapist is one of the protagonists in the therapeutic process. There are many efforts aimed at studying the factors that influence the establishment of a good therapeutic Alliance between therapist and patient that allows achieving a good psychotherapeutic outcome. This paper delves into the characteristics, abilities and actions of the psychotherapist in the context of the of the psychotherapeutic process. Some principles and challenges are proposed to contribute and promote continuous improvement in our professional practice as psychotherapists.

Keywords: Psychotherapist, Therapeutic Process, Therapeutic Alliance, Therapist's Personal Style.
\end{abstract}




\section{Introducción}

El presente artículo se basa en la presentación que se realizó en las I Jornadas Nacionales de la Asociación Española de Psicoterapias Cognitivas el 1 de febrero de 2019. De modo que este artículo es una adaptación en texto de buena parte de la exposición oral que se presentó en dichas Jornadas sobre las competencias del psicoterapeuta.

Son bastantes los autores que han propuesto distintos planteamientos teóricoprácticos respecto a las competencias de los psicoterapeutas (Corbella y FernándezÁlvarez, 2006). No cabe decir que con la aparición de los psicólogos generales sanitarios se reabrió la reflexión acerca de las competencias de los psicólogos en general y la de los psicólogos con distintos niveles de formación y especialización (PGS, especialistas en psicología Clínica, psicoterapeutas). Las regulaciones y acreditaciones de las diferentes profesiones sanitarias vinculadas a la psicología clínica y a la psicoterapia han generado tensiones entre los colectivos en defensa de sus intereses. En la actualidad hay múltiples profesiones vinculadas al mundo de la psicoterapia (psicólogos, psiquiatras, psicólogos especialistas en psicología clínica, psicólogos generales sanitarios, etc.) y prefiero evitar las disputas internas (sin desmerecer su legítima importancia) para destacar aquello que une a los distintos profesionales. Lejos de pretender contribuir a clarificar los límites de las competencias entre los distintos colectivos de profesionales implicados en la ayuda psicológica de los demás, me centraré exclusivamente en discernir sobre aquellas competencias, acciones y/o habilidades del profesional (psicoterapeuta, etc.) que sabemos que contribuyen de forma significativa en el desempeño del proceso terapéutico de ayuda.

\section{Proceso Terapéutico}

La práctica profesional de la psicoterapia no consiste en la mera aplicación de unas técnicas surgidas de un conocimiento teórico, sino que supone entre otras cosas el encuentro entre dos (o más) personas con sus características idiosincrásicas. Son muchos los esfuerzos dirigidos a estudiar los factores que influyen en el establecimiento de una buena relación entre psicoterapeuta y paciente que permita lograr un buen resultado psicoterapéutico (Corbella, 2019). En las últimas décadas se han llevado a cabo muchas investigaciones con el objetivo de estudiar los factores que puedan explicar la mejora de los pacientes en un proceso terapéutico. Uno de los factores que ha resultado ser determinante para la eficacia de la terapia es la calidad de la relación terapéutica (Corbella y Botella, 2003; Norcross y Lambert, 2018). Gelso y Carter $(1985,1994)$ definieron la relación terapéutica como "los sentimientos y actitudes que cada uno de los participantes tiene hacia el otro y la manera en que éstos son expresados” (Gelso y Carter, 1985, p. 159). Dentro del marco de la relación entre terapeuta y paciente se ha prestado especial atención al concepto de alianza terapéutica usado por primera vez por Greenson (1967).

Podemos entender la psicoterapia como un diálogo colaborativo entre el 
paciente y el terapeuta destinado a facilitar y promover el proceso de cambio. De modo que la psicoterapia consiste en la coconstrucción del diálogo fruto del encuentro entre el paciente y el terapeuta. Las posiciones subjetivas del paciente y las del terapeuta configuran las características de la relación terapéutica que se articula en las conversaciones durante la psicoterapia. Por lo tanto, la interacción entre la posición subjetiva del paciente y la del terapeuta influye fuertemente en el proceso de la terapia. Las características del terapeuta que configuran su identidad y su estilo personal, y los posicionamientos del paciente son los principales ingredientes influyentes en el desarrollo de la relación terapéutica y del trabajo terapéutico.

Se considera que la alianza terapéutica es una construcción conjunta entre paciente y terapeuta, de modo que las expectativas, las opiniones, las construcciones que ambos van desarrollando respecto al trabajo que están realizando, la relación establecida y la visión del otro resultan relevantes para el establecimiento de la alianza terapéutica, así como la alianza modula la relación (Corbella, 2019).

Las investigaciones con el objetivo de estudiar la relación entre la alianza terapéutica y la eficacia de la psicoterapia conforman un cuerpo considerable de publicaciones (véase por ejemplo, Barnicot, Katsakou, Bhatti, Savill, Fearns, y Priebe, 2012; Horvath, Del Re, Flückiger y Symonds, 2011; Weck, Grikscheit, Jakob, Höfling y Stangier, 2015). La mayoría de los estudios encuentran una relación significativa entre la alianza y el resultado final de la psicoterapia (Horvath y Symond, 1991; Horvath, 2001; Luborsky, 2000; Corbella y Botella, 2003; Horvath et al., 2011, Corbella, 2019).

En las últimas dos décadas han sido muchas las investigaciones centradas en la alianza terapéutica con el propósito de avanzar en su conocimiento y analizar las relaciones de la alianza con distintos elementos del proceso terapéutico así como de los resultados. Norcross y Wampold (2011) realizaron una serie de metanálisis sobre la efectividad de diferentes aspectos de la relación terapéutica aportando recomendaciones sobre las relaciones de terapia basadas en la evidencia. Encontraron que varios elementos de relación habían demostrado ser efectivos como la alianza en psicoterapia individual, la alianza en psicoterapia juvenil, la alianza en terapia familiar, la cohesión en terapia de grupo, la empatía, la recopilación de comentarios de clientes (Norcross y Wampold, 2011). También destacaban como elementos probablemente efectivos el consenso de objetivos, la colaboración y la consideración positiva.

Buena muestra de los desarrollos en el campo del estudio de la relación terapéutica la encontramos en que unos años más tarde Norcross y Lambert (2018) concluyeron que décadas de evidencia de investigación y experiencia clínica convergen y permiten afirmar que la relación de la psicoterapia hace contribuciones sustanciales y consistentes al resultado independientemente del tipo de tratamiento (Corbella, 2019). Norcross y Lambert (2018) describen los elementos de la relación que son demostrablemente efectivos, los probablemente efectivos y los prometedores, pero con investigación insuficiente para afirmarlo. 
Eficacia demostrada: La alianza en terapia individual, alianza en psicoterapia con jóvenes, alianza en terapia familiar, la cohesión en terapia de grupo, la empatía, la recopilación y receptividad a los comentarios del cliente, la colaboración, consenso de objetivos, afirmación y respeto positivo.

Aspectos probablemente eficaces: La congruencia y autenticidad, la reparación de rupturas de la alianza, y la gestión de la contratransferencia, relación real, expresión emocional, promover expectativas positivas, promover la credibilidad del tratamiento.

Prometedores, pero no hay pruebas suficientes para concluir que son eficaces: Autorrevelación e inmediatez.

Se ha cifrado entre un $6 \%$ y un $18 \%$ la variancia de la mejora del paciente que es explicada por variables del terapeuta (e.g., Lutz, Leon, Martinovich, Lyons y Stiles, 2007) por lo que cada vez son más los esfuerzos destinados a estudiar las características de los terapeutas que facilitan el desarrollo de la alianza y la obtención de resultados positivos en psicoterapia (Corbella, 2019).

\section{El psicoterapeuta como variable relevante e influyente en el proceso}

Las actitudes terapéuticas propuestas por Rogers (1957) despertaron gran interés en investigaciones posteriores, que buscaron las características y las conductas del terapeuta beneficiosas para el resultado terapéutico (Orlinsky y Howard, 1986; Patterson y Forgatch, 1985). La empatía y la calidez del terapeuta son dos de las cualidades que la mayoría de los investigadores coinciden en considerar relevantes (pero no suficientes) para establecer una buena alianza terapéutica (Bachelor y Horvath, 1999; Ackerman y Hilsenroth, 2003). Henry y Strupp (1994) encontraron que comportamientos de exploración y valoración por parte del terapeuta facilitaban la alianza con el paciente, mientras que la baja valoración perjudicaba a la alianza.

Ackerman y Hilsenroth (2003) encontraron un conjunto de actitudes y valores (curiosidad, flexibilidad, respeto, honestidad, sinceridad y cordialidad) que influyen positivamente en el establecimiento de la alianza terapéutica. Nienhuis et al. (2018) encontraron el papel relevante de la empatía y la autenticidad del terapeuta en la construcción de la alianza terapéutica con el paciente.

Algunos estudios constatan que la relación entre la experiencia del terapeuta y la alianza presenta no es significativa (Dunkle y Friedlander, 1996; Hersoug, Hoglend, Monsen y Havik, 2001; Kivlighan, Patton y Foote, 1998) a pesar de que otros estudios han encontrado relaciones entre la experiencia del terapeuta y la calidad de la alianza terapéutica, especialmente en el componente de acuerdo en los objetivos (MallincKrodt y Nelson, 1991). Tschuschke et al. (2014) encontraron, entre otros resultados, una relación positiva entre la experiencia del terapeuta y la alianza terapéutica.

La relación entre la formación del psicoterapeuta y la alianza y/o resultado terapéutico ha sido un interrogante "histórico" en el campo de la investigación en psicoterapia. MallincKrodt y Nelson (1991) vieron que la formación de los tera- 
peutas influía positivamente en el establecimiento de la alianza terapéutica. Por otro lado, Hersoug et al. (2001) identificaron que el entrenamiento y la formación de los terapeutas eran variables que estaban relacionadas positivamente con la alianza terapéutica cuando esta era evaluada por los terapeutas, pero no cuando la alianza era evaluaba por el paciente. Horvath (2001) encontró que la relación entre el nivel de formación del terapeuta y la calidad de la alianza es inconsistente. Resulta evidente que los programas formativos que tienen como objetivo el trabajo de acciones/habilidades y la adquisición de competencias directamente vinculadas a la alianza, la cohesión en grupos, empatía, consenso de objetivos, entre otros (Norcross y Lambert, 2018) son facilitadoras y promotoras de la mejora competencial de los psicoterapeutas y previsiblemente de sus resultados (Consoli, Fernández-Álvarez y Corbella, 2017).

Safran, Muran y Samstag (1994) encontraron que se favorecía a la alianza terapéutica cuando el terapeuta tenía la capacidad de aceptar parte de la responsabilidad por sus rupturas relacionales en terapia y cuando se metacomunicaba con el paciente en relación a posibles malentendidos. También se han estudiado algunas de las actividades técnicas y las acciones del terapeuta durante las sesiones relacionándolas con el desarrollo de la alianza. Se localizó que cuando el terapeuta centra su actividad en el aquí y ahora y la orienta temáticamente se favorece la alianza (Kivlighan y Schmitz, 1992). Por otro lado, Crowley (2001) encontró que la mentalidad psicológica del terapeuta y sus habilidades sociales correlacionaban positivamente con la alianza terapéutica.

Algunas investigaciones han destacado el papel de las habilidades interpersonales facilitadoras de los terapeutas en el establecimiento de la alianza terapéutica con los pacientes y en los resultados positivos alcanzados en psicoterapia (Anderson, Crowley, Himawan, Holmberg y Uhlin, 2016; Anderson, McClintock, Himawan, Song y Patterson, 2016; Anderson, Ogles, Patterson, Lambert y Vermeersch, 2009; Schöttke, Flückiger, Goldberg, Eversmann, y Lange, 2017).

En el estudio de Anderson et al. (2009), los terapeutas completaron un cuestionario de habilidades sociales y luego vieron varios videos de clientes desafiantes, después de lo cual registraron su respuesta terapéutica. Sus respuestas fueron codificadas según lo que Anderson y sus colegas denominaron Habilidades Interpersonales Facilitadoras (HIF), que incluían la fluidez verbal, expresión emocional, persuasión, esperanza, calidez, empatía, alianza y enfoque del problema. Resultó que las calificaciones de HIF predecían los resultados de los terapeutas: los clientes de los terapeutas que obtuvieron puntuaciones FIS más altas en esta prueba de desafío se beneficiaron más de la terapia que los clientes de terapeutas con puntuaciones HIF más bajas.

Los terapeutas efectivos tienen un conjunto de habilidades terapéuticas que utilizan con los pacientes, especialmente los pacientes desafiantes. También se ha encontrado que los terapeutas efectivos cuestionan su nivel de habilidad (como auto-duda profesional) y dedican esfuerzos fuera de la consulta tratando de mejorar 
su práctica profesional (Wampold, Baldwin, Holtforth e Imel, 2017). También hay características y acciones de los terapeutas que no parecen estar relacionadas con su efectividad, incluida la edad, el sexo, la profesión, orientación teórica, grado de adhesión a un manual de tratamiento, o la competencia calificada que brinda un tratamiento en particular con un cliente en particular (Corbella, 2019).

Las características y las habilidades/acciones de los psicoterapeutas efectivos serían las siguientes (Wampold et al., 2017; Anderson et al., 2009): a) Formación de alianzas en un rango amplio de pacientes y la capacidad de reparar rupturas de alianzas, b) Fluidez verbal, c) Calidez y empatía, d) Expresión emocional, e) Persuasión, f) Optimismo, g) Enfoque del problema, h) Entrega de un tratamiento convincente, i) Duda profesional y tiempo dedicado a mejorar.

La relevancia de estudiar la relación entre las intervenciones del terapeuta y las variables del cliente es lo que impulsó la Selección Sistemática de Tratamientos (SST) propuesta por Beutler (Beutler, Clarkin y Bongar, 2000; Beutler, et al., 2016). Dos de las dimensiones que configuran la SST son: (a) la directividad del terapeuta relacionada con la resistencia del paciente y (b) la tendencia del terapeuta a dirigir la atención a la acción o al insight del paciente relacionada con el estilo de afrontamiento del paciente. Así, Beutler considera que, por ejemplo, un paciente resistente y con un estilo de afrontamiento internalizador se beneficiaría más de un terapeuta poco directivo y dirigido al insight (Corbella, 2019).

Rubino, Barker, Roth y Fearon (2000) estudiaron la relación entre los estilos de apego y la decisión del terapeuta de romper la alianza, encontrando que los terapeutas más ansiosos tienden a responder con menor empatía, particularmente con los pacientes ansiosos y seguros. Diferentes investigaciones han encontrado que el apego inseguro del terapeuta puede afectar negativamente la alianza terapéutica en clientes y destacan la importancia de considerar la interacción entre el apego del cliente y el terapeuta así cómo estas interacciones influyen en la alianza terapèutica (Bucci, Seymour-Hyde, Harris y Berry, 2016; Degnan, Seymour, Harris y Berry, 2016).

Beutler et al. (2000, 2016), entre otros, han constatado la necesidad de estudiar las variables del paciente en relación con las del tratamiento. También las variables del terapeuta deben ser estudiadas en relación con las del cliente (Hill, 2006; Corbella y Botella, 2004). De aquí surgieron iniciativas de estudios sobre la interacción entre terapeuta y paciente. Si bien la fuerza de la alianza entre terapeuta y paciente es el mejor predictor del éxito de la terapia, no hay una única variable del terapeuta o del paciente que por sí misma, y con independencia de las otras, sea decisiva en cuanto a la determinación de la fuerza de dicha alianza (véase Corbella y Botella, 2004). Uno de los estudios sobre la interacción Terapeuta-Paciente es el Proyecto Barcelona Buenos Aires que se llevó a cabo conjuntamente entre la Fundación Aiglé de Buenos Aires y la Facultat de Psicologia, Ciències de l'Educació i de l'Esport Blanquerna de la Universitat Ramon Llull de Barcelona. De este estudio se extrajeron algunos principios útiles para el trabajo psicoterapéutico (Corbella 
2003; Corbella et al., 2009). Los principios fundamentales surgidos del Proyecto Barcelona-BsAs fueron los siguientes (Corbella y Botella, 2004):

1. La derivación o asignación de un paciente a un terapeuta u otro es muy importante para el proceso y resultado de la psicoterapia.

2. En la asignación del paciente a un terapeuta se debe tener en cuenta la resistencia del paciente y el estilo del terapeuta. De modo que es recomendable que aquellos pacientes más resistentes se asignen a terapeutas con un estilo poco pautado y poco directivo.

3. La resistencia del paciente se debe tener en cuenta en el proceso de evaluación inicial y el terapeuta debe trabajar con ella para beneficiar al paciente. La resistencia no es una dificultad del paciente sino que es un reto para el trabajo conjunto entre paciente y terapeuta.

4. El terapeuta debe conocer su propio estilo personal como terapeuta para facilitar la optimización de sus recursos y habilidades personales, así como para mejorar aquellos aspectos que le suponen alguna dificultad.

5. El terapeuta debe adaptar su modo de comunicación, su estilo terapéutico, a las características del paciente (especialmente la resistencia) en la medida que le sea posible. De modo que, si el paciente es resistente, el terapeuta debe intentar adaptar su estilo para resultar ser poco pautado y poco directivo.

6. El esfuerzo del terapeuta en adaptar su estilo a las características del paciente se debe reducir si supone una incomodidad por la pérdida de coherencia teórica del terapeuta al emplear los diferentes procedimientos o estrategias comunicativas. Aun así, debe ser consciente de qué tipo de estilo terapéutico puede ser más conveniente para cada tipo de paciente.

7. La formación de psicoterapeutas debe profundizar en el estilo personal del terapeuta, fomentar la autoreflexión y el estilo flexible en terapia.

8. Durante el proceso psicoterapéutico y especialmente en las primeras ocho sesiones, el terapeuta debe prestar atención a trabajar hacia el establecimiento de una buena alianza terapéutica con el paciente.

En Corbella (2019) se profundiza sobre la relación entre el Estilo Personal del Terapeuta y el desarrollo de la alianza terapéutica.

Se ha puesto de manifiesto la importancia de la interacción entre el terapeuta y el paciente, pero todavía quedan muchas preguntas interesantes esperando ser abordadas con planteamientos sugerentes. Algunas de estas preguntas guardan relación acerca de los factores o las habilidades del terapeuta que explican que se establezca una buena relación terapéutica con el paciente y que faciliten un proceso psicoterapéutico exitoso. La base teórica de la Teoría de la Mente nos propone elementos de reflexión para plantear respuestas a algunas de las preguntas planteadas (ver Corbella et al., 2009).

El terapeuta como persona y miembro de su familia ha experimentado distintas relaciones y suma diferentes vivencias que van configurando sus posicionamientos 
relacionales y sus características particulares que le dan identidad y que influyen en la concepción y desarrollo de su actividad profesional (Corbella y FernándezÁlvarez, 2006). La interacción que se da desde un principio entre la persona del terapeuta y su entorno relacional (como el contexto familiar) influye fuertemente en el desarrollo de distintos niveles de la teoría de la mente que resultan especialmente importantes para los psicoterapeutas.

Se puede plantear que la teoría de la mente o las distintas habilidades mentalistas son los fundamentos de la empatía (en el contexto terapéutico) así como los precursores de actitudes terapéuticas y de distintos recursos que facilitan no únicamente el establecimiento de la relación terapéutica sino también la intervención más conveniente adaptada al paciente.

Resulta evidente la relevancia que tiene para el terapeuta el proceso cognitivo que permite a las personas ser capaces de predecir estados mentales de uno mismo y de los demás, lo que facilita anticipar y modificar comportamientos propios y ajenos. La habilidad de inferir estados mentales (como pensamientos, deseos, intenciones, etc.) en otra persona, posibilita utilizar la información para interpretar (a) ¿qué dicen?; (b) ¿por qué lo dicen?; y (c) predecir qué puede pasar a continuación. Parece difícil imaginar que un psicoterapeuta pudiera trabajar como tal sin disponer de unas mínimas habilidades propias de la teoría de la mente (Corbella et al, 2009).

Un trabajo de formación de psicoterapeutas basado no solo en la adquisición de competencias vinculadas a los conocimientos psicopatológicos y de técnicas de intervención sino también basados fundamentalmente en el trabajo del estilo personal del terapeuta y la optimización de las habilidades de la terapia de la mente sirve para mejorar nuestra práctica terapéutica.

La adquisición de las habilidades de la teoría de la mente y la complejidad de su uso se va configurando a lo largo de nuestro desarrollo y va proporcionando unas características singulares a nuestra identidad y nuestras interacciones con las demás personas. En el ámbito de la psicoterapia planteamos la influencia consistente que ejerce la teoría de la mente del terapeuta sobre el desarrollo del estilo personal del terapeuta. Uno de los aspectos del estilo terapéutico más significativamente influido por las habilidades de la teoría de la mente es la flexibilidad versus rigidez del terapeuta (Corbella et al., 2009). La función instruccional tiene un papel moderador sobre las otras funciones del estilo del terapeuta, de modo que un terapeuta más flexible tendrá más facilidad para adaptar su estilo a las necesidades de cada paciente (Corbella, 2003).

El desarrollo óptimo de la teoría de la mente del terapeuta permite (facilita) la habilidad terapéutica de entender como el paciente procesa la información, piensa, siente y actúa así como la habilidad de anticipar la conducta del paciente y sus posibles reacciones ante ciertas situaciones. Estas habilidades desempeñan una función esencial no únicamente para poner en acción la empatía terapéutica sino para autorregular la interacción del terapeuta con su paciente (Corbella et al., 2009).

Todas las habilidades propias de la teoría de la mente influyen sobre el estilo 
personal del terapeuta y facilitan que el terapeuta adapte su estilo y sus intervenciones a las características del paciente. De modo que estas habilidades resultan esenciales para desarrollo de una adecuada alianza terapéutica con el paciente y para encontrar las estrategias más apropiadas para ayudar al paciente.

Podemos plantear un conjunto de recomendaciones, retos y aspiraciones para terapeutas basadas, en gran parte, en las características, competencias y acciones habituales de los psicoterapeutas eficaces (basado en: Anderson et al., 2009, 2016; Corbella et al., 2009; Schöttke et al., 2017; Wampold et al., 2017; citados en Corbella, 2019).

- Mantener una práctica reflexiva como camino para la mejora constante (autocuestionamiento).

Hacer esfuerzos en cultivarcierta introspección para facilitar un posicionamiento de la experiencia más analítico permite que el ejercicio profesional no consista en la mera acumulación pasiva de experiencias.

- Ser competente en leer el estado afectivo y mental del paciente. Habilidades en teoría de la mente, metacognición.

Disponer de las habilidades en la teoría de la mente facilita cualquier interacción personal y como psicoterapeutas nos ayuda de forma determinante en la comprensión del paciente y en el establecimiento (y desarrollo) de la alianza terapéutica del proceso de ayuda.

- Mantener un Estilo Personal flexible que facilite la adaptación del terapeuta a las necesidades del paciente en función de sus características y circunstancias a lo largo del proceso terapéutico.

Incrementar los grados de flexibilidad del estilo personal del terapeuta permite ajustar con mayor facilidad el estilo de la intervención terapéutica a las características del paciente. La flexibilidad del terapeuta facilita la modulación y el cambio del propio estilo personal.

- Dominar las habilidades interpersonales.

Entendiendo el proceso terapéutico como la interacción de ayuda entre el paciente y el psicoterapeuta se hace evidente que las habilidades relacionales del profesional juegan un papel determinante.

- Ser capaz de establecer una alianza de trabajo fuerte y colaborativa con un amplio abanico de pacientes.

Adaptarse a las características y necesidades del paciente resulta imprescindible para establecer una buena relación terapéutica. Disponer de las habilidades que permiten fortalecer el vínculo emocional positivo y la alianza terapéutica con el paciente es uno de los aspectos característicos de los psicoterapeutas más eficaces.

- Tener fluidez verbal, riqueza léxica y precisión en el uso del lenguaje para expresar lo que se pretende.

La comunicación del terapeuta es un elemento esencial en el proceso terapéutico y disponer de recursos de expresión verbal y no verbal que permitan dotar a la comunicación de los detalles y precisión necesaria facilita la funcionalidad y 
el objetivo de toda acción comunicativa del psicoterapeuta.

- Saber modular tus propias reacciones afectivas.

La autorregulación afectiva del psicoterapeuta es un aspecto facilitador en la interacción con el paciente al incrementar la toma de consciencia de las propias emociones por parte del terapeuta y así gestionarlas sin que estas influyan o sesguen negativamente el proceso psicoterapéutico que debe pivotar sobre el paciente.

- Ser competente en activar/implicar a los pacientes.

La implicación y la motivación de cualquier persona tiene una gran relevancia en un proceso de cambio. Movilizar el afecto del interlocutor activando su implicación en la consecución de los objetivos propuestos tiene un peso específico en el proceso terapéutico.

- Ser competente en transmitir cordialidad, empatía y comprensión.

Algunos de los puntos comentados anteriormente podrían englobar éste. Sin embargo, no sobra enfatizar la necesaria contención empática y la comprensión de como el paciente vive su problemática para facilitar el adecuado proceso de ayuda.

- Ser competente en centrarse en el paciente y no en uno mismo.

Evitar la primacía de la visión centrada en las hipótesis, expectativas y experiencias personales del psicoterapeuta permite centrar la atención en la experiencia ideográfica del paciente como elemento facilitador del proceso terapéutico.

- Ser competente en atribuir las mejoras al trabajo del paciente.

Del punto anterior se desprende éste que no deja de centrar la mirada, el protagonismo y la responsabilidad del éxito terapéutico a las acciones y esfuerzos del paciente. Ceder todo el protagonismo al paciente en la atribución de responsabilidades de cambio cuando se ha logrado alcanzar los objetivos facilita los beneficios de un buen resultado al finalizar el proceso terapéutico.

- Ser permeables y abiertos a recibir feedback del progreso de los pacientes para plantear cambios si no mejora (monitorear resultados).

Huir de un hermetismo estanco del terapeuta en la valoración de la evolución de cada proceso terapéutico y ser receptivos a las distintas fuentes que pueden proporcionar información relevante para mejorar nuestra comprensión e intervención psicoterapéutica. Conviene una mente abierta que se enriquezca de la información disponible, de los cuestionarios/tests u otros instrumentos que contribuyen sin duda a incrementar la información acerca del paciente y del desarrollo del proceso terapéutica.

- Saber aplicar los principios de la STS (Beutler et al., 2000, 2016) y la adaptación de las intervenciones y el propio Estilo Personal del Terapeuta a las características del paciente en función de las dimensiones de la STS.

La STS consiste en la aplicación de unos principios en las que se ajustan las intervenciones a las características del paciente según unas variables específicas (Beutler et al., 2016): a) Modular y adaptar la intensidad del tratamiento/intervención al nivel de deterioro funcional del paciente; b) Dirigir, preferentemente, la intervención a la acción o al insight según el estilo de afrontamiento del paciente; 
c) Adaptar el nivel de directividad de la intervención al nivel de resistencia del paciente; d) Ajustar la activación o reducción del afecto según el nivel de malestar Subjetivo del paciente; e) Adaptar, regular y prever la duración del proceso terapéutico a partir de información acerca del apoyo social del paciente.

- Ser convincente al proporcionar el tratamiento al paciente.

La manera en la que los psicoterapeutas desempeñan su función está fuertemente vinculada con el estilo terapéutico. La implicación y el convencimiento del terapeuta en su propia intervención resultan relevante para potenciar su efecto.

- Gestionar el conocimiento adquirido manteniendo la humildad para seguir mejorando y potenciando el aprendizaje en distintas áreas.

Una actitud humilde y constructiva facilita la apertura a nuevos aprendizajes que contribuyen a la mejora de nuestra práctica. Mantener la mente abierta a los muchos cambios que se están produciendo y a los nuevos conocimientos que van apareciendo facilita la adaptación de la práctica profesional del psicoterapeuta a las nuevas circunstancias que como sociedad nos vamos encontrando.

Plantear los puntos anteriores como retos y objetivos para marcarnos el rumbo de la mejora personal como psicoterapeutas siempre será más constructivo y saludable que formularlo como requisitos indispensables para la correcta acción terapéutica ya que nos supondría una fuente de ansiedad añadida.

Las competencias y acciones expuestas anteriormente no pretenden configurar una lista exhaustiva y limitada, sino que más bien tiene la intención de abrir foco y señalar solo algunas de las muchas que están por incorporar y que incluso pueden tener matices diferenciados según el estilo personal de cada terapeuta. Lo planteado en el artículo no pretende ser nada más que una reflexión sobre las competencias y habilidades del terapeuta en el contexto del proceso terapéutico tal y como se expuso en la presentación en las I Jornadas Nacionales de la Asociación Española de Psicoterapias Cognitivas el 1 de febrero de 2019.

\section{Referencias Bibliográficas}

Ackerman, S. J. y Hilsenroth, M. J. (2003). A review of therapist char-acteristics and techniques positively impacting the therapeutic alliance. Clinical Psychology Review, 23(1),1-33. https://doi.org/10.1016/ S0272-7358(02)00146-0

Anderson, T., Crowley, M., Himawan, L., Holmberg, J. y Uhlin, B. (2016). Therapist facilitative interpersonal skills and training status: Arandomized clinical trial on alliance and outcome. Psychotherapy Research, 26(5), 511529. https://doi.org/10.1080/10503307.2015.1049671

Anderson, T., McClintock, A. S., Himawan, L., Song, X. y Patterson, C. L. (2016). A prospective study of therapist facilitative interpersonal skills as a predictor of treatment outcome. Journal of Consulting and Clinical Psychology, 84(1), 57-66. https://doi.org/10.1037/сcp0000060

Anderson, T., Ogles, B. M., Patterson, C. L., Lambert, M. J. y Vermeersch, D. A. (2009). Therapist effects: Facilitative interpersonal skills as a predictor of therapist success. Journal of Clinical Psychology, 65(7), 755-768. https://doi.org/10.1002/jclp.20583

Bachelor, A. y Horvath, A. (1999). The therapeutic relationship. En M. A. Hubble, B. L. Duncan y S. D. Miller (Eds.), The heart and soul of change: What works in therapy (pp. 133-178). Washington, DC: American Psychological Association. 
Barnicot, K., Katsakou, C., Bhatti, N., Savill, M., Fearns, N. y Priebe, S. (2012). Factors predicting the outcome of psychotherapy for borderline personality disorder: a systematic review. Clin Psychol Rev, 32(5), 400412. https://doi.org/10.1016/j.cpr.2012.04.004

Beutler, L. E., Clarkin, J. F. y Bongar, B. (2000). Guidelines for the systematic treatment of the depressed patient. Nueva York, NY: Oxford University Press. https://doi.org/10.1093/acprof:oso/9780195105308.001.0001

Beutler, L. E., Someah, K., Kimpara, S. y Miller, K. (2016). Selecting the most appropriate treatment for each patient. International Journal of Clinical and Health Psychology, 16(1), 99-108. https://doi.org/10.1016/j. ijchp.2015.08.001

Bucci, S., Seymour, H. A., Harris, A. y Berry, K. (2016). Client and therapist attachment styles and working alliance. Clinical Psychology \& Psychotherapy, 23(2), 155-165. https://doi.org/10.1002/cpp.1944

Consoli, A. J., Fernández-Álvarez, H. y Corbella, S. (2017). The training and development: A lifespan perspective. En A. J., Consoli, L. E., Beutler y B. Bongar (Eds.), Comprehensive textbook of psychotherapy: Theory and practice ( $2^{\mathrm{a}}$ ed., pp. 462-479). Nueva York, NY: Oxford University Press.

Corbella, S. (2003). Compatibilidad entre el Estilo Personal del Terapeuta y el Perfil Personal del Paciente (Tesis doctoral, Universidad de Ramon Llull, Barcelona, España). Recuperada de: https://dialnet.unirioja. es/servlet/tesis?codigo=220061

Corbella, S. (2019). El Estilo Personal del Terapeuta y la Alianza Terapéutica. En H. Fernández-Álvarez y F. García (Eds.), El Estilo Personal del Terapeuta (pp. 57-90). Buenos Aires, Argentina: Editorial Polemos.

Corbella, S., Balmaña, N., Fernández-Álvarez, H., Saúl, L. A., Botella, L. y García, F. (2009). Estilo Personal del Terapeuta y teoría de la mente. Revista Argentina de Clínica Psicológica, 18(2), 125-133. Recuperado de: https://www.redalyc.org/pdf/2819/281921792003.pdf

Corbella, S. y Botella, L. (2003). La alianza terapéutica: historia, investigación y evaluación. Revista Anales de Psicología, 19(2), 205-221. Recuperado de: https://revistas.um.es/analesps/article/view/27671

Corbella, S. y Botella, L. (2004). Investigación en Psicoterapia: Proceso, Resultado y Factores Comunes. Madrid, España: Vision Net.

Corbella, S. y Fernández-Álvarez, H. (2006). El terapeuta, posiciones, actitudes y estilos personales. En L. Botella (Ed.), Construcciones, narrativas y relaciones: Aportaciones constructivistas y construccionistas a la psicoterapia (pp. 299-340). Barcelona, España: Edebe.

Corbella, S., Fernández-Álvarez, H., Saúl, L. A., García, F., y Botella, L. (2008). Estilo Personal del Terapeuta y Dirección de Intereses. Apuntes De Psicología, 26(2), 281-289. Recuperado de: http://www.apuntesdepsicologia.es/index.php/revista/article/view/261/263

Crowley, M. J. (2001). Patient and therapist pre-treatment characteristics as predictors of the therapeutic alliance (Tesis doctoral no publicada). Ohio University, Ohio, OH.

Degnan, A., Seymour, H. A., Harris, A. y Berry, K. (2016). The role of therapist attachment in alliance and outcome: A systematic literature review. Clinical Psychology \& Psychotherapy, 23(1), 47-65. https://doi. org/10.1002/cpp.1937

Dunkle, J. H. y Friedlander, M. L. (1996). Contribution of therapist experience and personal characteristics to the working alliance. Journal of Counseling Psychology, 43(4), 456-460. https://psycnet.apa.org/ doi/10.1037/0022-0167.43.4.456

Gelso, C. J. y Carter, J. A. (1985). The Relationship in counseling and psychotherapy: Components, consequences and theoretical antecedents. The Counseling Psychologist, 13(2), 155-244. https://doi. org/10.1177\%2F0011000085132001

Gelso, C. J. y Carter, J. A. (1994). Components of the psychotherapy Relationship: Their interaction and unfolding during treatment. Journal of Counseling Psychology, 41(3), 296-306. https://doi.org/10.1037/00220167.41.3.296

Greenson, R. R.(1967). Technique and practice of psychoanalysis. Nueva York, NY: International University Press.

Henry, W. P. y Strupp, H. H. (1994). The therapeutic alliance as interpersonal process. En A. O. Horvath y L. S. Greenber (Eds.), The working alliance: Theory, research, and practice (pp. 51-84). Nueva York, NY: John Wile.

Hersoug, A. G., Hoglend, P., Monsen, J. T. y Havik, O. E. (2001). Quality of working alliance in psychotherapy: Therapist variables and patient/therapist similarity as predictors. Journal of Psychotherapy Practice and Research, 10(4), 205-216.

Hill, C. (2006). Introduction to special section on therapist effects. Psychotherapy Research, 16(2), 143-144. https://doi.org/10.1080/10503300500470583

Horvath, A. O. (2001). The alliance. Psychotherapy: Theory, Research, Practice, Training, 38(4), 365-372. https:// doi.org/10.1037/0033-3204.38.4.365 
Horvath, A. O., Del Re, A. C., Flückiger, C. y Symonds, D. (2011). Alliance in Individual Psychotherapy. Psychotherapy, 48(1), 9-16.

Horvath, A. O. y Symonds, B. D. (1991). Relation between alliance and outcome in psychotherapy: A metaanalysis. Journal of Counseling Psychology, 38(2), 139-149. https://doi.org/10.1037/0022-0167.38.2.139

Kivlighan, D. M. Jr., Patton, M. J. y Foote, D. (1998). Moderating effects of client attachment on the counselor experience-working alliance relationship. Journal of Counseling Psychology, 45(3), 274-278. https://doi. org/10.1037/0022-0167.45.3.274

Kivlighan, D. M. y Schmitz, P. J. (1992). Counselor technical activity in cases with improving working alliances and continuing-poor working alliances. Journal of Counseling Psychology, 39(1), 32-38. https://doi. org/10.1037/0022-0167.39.1.32

Luborsky, L. (2000). A pattern-setting therapeutic alliance study revisited. Psychotherapy research, 10(1), 17-29. https://doi.org/10.1080/713663591

Lutz, W., Leon, S. C., Martinovich, Z., Lyons, J. S. y Stiles, W. B. (2007). Therapist effects in outpatient psychotherapy: A three-level growth curve approach. Journal of Counseling Psychology, 54(1), 32-39. https:// doi.org/10.1037/0022-0167.54.1.32

Mallinckrodt, B. y Nelson, M. L. (1991). Counselor training level and the formation of the psychotherapeutic working alliance. Journal of Counseling Psychology, 38(2), 133-138. https://doi.org/10.1037/0022-0167.38.2.133

Muran, J. C., Segal, Z. V., Samstag, L. W. y Crawford, C. E. (1994). Patient pretreatment interpersonal problems and therapeutic alliance in short-term cognitive therapy. Journal of Consulting and Clinical Psychology, 62(1), 185-190. https://doi.org/10.1037/0022-006X.62.1.185

Nienhuis, J. Owen,J., Valentine J., Winkeljohn Black, V., Halford, T., Parazak, S., ... Hilsenroth, M. (2018). Therapeutic alliance, empathy, and genuineness in individual adult psychotherapy: A meta-analytic review. Psychotherapy Research, 28(4), 593-605. https://doi.org/10.1080/10503307.2016.1204023

Norcross, J. C. y Lambert, M. J. (2018). Psychotherapy relationships that work III. Psychotherapy, 55(4), 303-315. http://dx.doi.org/10.1037/pst0000193

Norcross, J. C. y Wampold, B. E. (2011). Evidence-based therapy relationships: Research conclusions and clinical practices. Psychotherapy, 48(1), 98-102. https://doi.org/10.1037/a0022161

Orlinsky, D. y Howard, K. (1986). Process and outcome in psychotherapy. En S. L. Garfield y A. E. Bergin (Eds.), Handbook of psychotherapy and behavior change ( $3^{\mathrm{a}}$ ed., pp. 311-381). Nueva York, NY: Wiley.

Patterson, G. R. y Forgatch, M. S. (1985). Therapist behavior as a determinant for client noncompliance: A paradox for the behavior modifier. Journal of Consulting and Clinical Psychology, 53(6), 846-851. https:// doi.org/10.1037/10412-016

Rogers, C. R. (1957). The necessary and sufficient conditions of therapeutic personality change. Journal of Consulting Psychology, 21(2), 95-103. https://doi.org/10.1037/h0045357

Rubino, G., Barker, C., Roth, T. y Fearon, P. (2000). Therapist empathy and depth of interpretation in response to potential alliance ruptures: The role of therapist and patient attachment styles. Psychotherapy Research, 10(4), 408-420. https://doi.org/10.1093/ptr/10.4.408

Safran, J. D., Muran, J. C. y Samstag, L. W. (1994). Resolving therapeutic alliance ruptures: A task analytic investigation. En A. O. Horvath y L. S. Greenberg (Eds.), The working alliance: Theory, research, and practice. Wiley series on personality processes (pp. 225-255). Nueva York, NY: John Wiley and Sons.

Schöttke, H., Flückiger, C., Goldberg, S. B., Eversmann, J. y Lange, J. (2017). Predicting psychotherapy outcome based on therapist interpersonal skills: A five-year longitudinal study of a therapist assessment protocol. Psychotherapy Research, 27(6), 642-652. https://doi.org/10.1080/10503307.2015.1125546

Tschuschke, V., Crameri, A., Koehler, M., Berglar, J., Muth, K., Staczan, P., ... Lutz, M. (2014). The role of therapists' treatment adherence, professional experience, therapeutic alliance, and clients' severity of psychological problems: Prediction of treatment outcome in eight different psychotherapy approaches. Preliminary results of a naturalistic study. Psychotherapy Research, 25(4), 420-434. https://doi.org/10.1 080/10503307.2014.896055

Wampold, B. E., Baldwin, S. A., Holtforth, M. G. e Imel, Z. E. (2017). What Characterizes Therapist Effects? En L. G. Castonguay y C. E. Hill (Eds.), How and why are some therapists better than others: Understanding therapist effects (pp. 37-53). Washington DC: American Psychological Association.

Weck, F., Grikscheit, F., Jakob, M., Höfling, V. y Stangier, U. (2015). Treatment failure in cognitive-behavioural therapy: therapeutic alliance as a precondition for an adherent and competent implementation of techniques. Br J Clin Psychol. 54(1), 91-108. https://doi.org/10.1111/bjc.12063 